\title{
Mechanical Properties of Al2219-TiC Metal Matrix Composites
}

\author{
Naresh Babu, Ch.Santosh Kumar Reddy
}

\begin{abstract}
Work turned into finished to explore and observe the mechanical residences of TiC fortified metal grid composite amalgam Al2219. In this investigation Al2219 compound taken because the important framework and particulate $\mathrm{TiC}$ as a fortification cloth for putting in a metal grid composite with mixing throwing method. For help steel framework composite material shifts from zero to 6 wt. In stage 2 wt\%. \%. For each composite, particulate fortification is warmed to a temperature of $600^{\circ} \mathrm{C}$ and spread into the vortex of liquid amalgam Al2219. Microstructural portrayal is finished by using utilizing an examining electron magnifying tool. Mechanical homes, for example, hardness, intense elasticity, yield high-quality and growth rate became assessed by means of ASTM norms. Further, examining electron microphotographs uncovered that there's a uniform dissemination of particulate $\mathrm{TiC}$ in Al2219 aggregate grid. Hardness, extreme rigidity and yield satisfactory builds wt. $\%$ Of TiC increment in the fundamental framework. Results likewise verified that as wt. \% Of aid increments, there may be an abatement in malleability.
\end{abstract}

Keywords Al2219 compound, TiC, mechanical homes, extreme rigidity, hardness, Stir throwing, steel framework composites

\section{INTRODUCTION}

Metal system composites are progressively turning out to be engaging substances for diminishing aspect avionics, vehicle organizations as a result of light weight, ease, simple production and frequently expanding solicitations of current advancement. Metal system composites are the blend of touchy base metal with hard refinement fabric and highlight starting late watched specialinterestbecauseoftheirspecificstrengthandspecific robustness at room or raised temperature. With the movement of cutting edge advancement, there is an everlasting enthusiasm for a reasonable, light weight more noteworthy tenaciously, strongerand control saving material inside the domain of room, flying device, impelled shield warrior streams and vehicle programming, aluminum network composites (AMC) decided utility in those locales $[1,2]$. Various predominant presentation strategies in which being utilized for the gathering of MMC materials as demonstrated with the guide of the type of base texture and the kind of help applied like mix tossing, press tossing, liquid metallic infiltration and wash co-articulation. Amongtheabove, mix tossing approach is the least intense and most proficient applied gadget is knownas'vortextechnique'orstircasting

machine' it's miles alluring an immediately final product of straightforwardness, least endeavor of managing,

Revised Manuscript Received on September 14, 2019.

Mr. Naresh Babu, Assistant Professors, Siddhartha Institute of Technology and Sciences,Narapally, Hyderabad, Telangana, India.

Mr.Ch.Santosh Kumar Reddy, Assistant Professors, Siddhartha Institute of Technology and Sciences,Narapally, Hyderabad, Telangana, India. versatility, greatest fiscally for good measured anticipated parts to be set up similarly as period of near web shaped fragments.

In light of improvement in development, there is progressed demandforaneconomical,lightweight,harder,strongerand control saving material inside the zone of room, air convey, security and vehicle application and aluminum cross section found bundles in these domains [3]. Aluminum compound strengthened with troublesome innovative flotsam and jetsam of $\mathrm{WC}, \mathrm{SiC}, \mathrm{Al} 2 \mathrm{O} 3, \mathrm{~B} 4 \mathrm{C}$ and graphite for surrounding a composite to recognize overhauls in mechanical living arrangements, as an occasion, hardness, more youthful's modulus, yield pleasant and outrageous flexibility of the MMCs, The composites finds utility in avionics and vehicle adventures [4]. Regardless of the truth that blend tossing is the most usually systems applied for the MMCs, wettability is the key issue related with mix tossing To beat the wettability issue happening for the most part, wetting administrator especially magnesium has been controlled by certain examiners.

AMCs with $\mathrm{SiC}$ on the grounds that the help atom, $\mathrm{SiC}$ has a major smidgen of breathing space of improving the living arrangements like low thickness, high acceptable, low warmth augmentation, high warmth conductivity, extreme hardness, high adaptable modulus, excellent warmth daze, restriction and nonexclusive substance inactivity. Need has been complemented for creating moderate Al-based MMCs with select hard and touchy fortresses

likeSiC,Al2O3,zircon,graphiteandmica[5].QiurongYang et al. Inspected that the moldable investigate, extending of the composites recommends a pointy increase from four.Five \% to thirteen.Five\% due towovencarbonfibers.Meanwhilethetensilestrengthofthe composite is expanded barely from $168 \mathrm{Mpa}$ to $202 \mathrm{MPa}$ comparedtothatofZL205Aalloy.Thegoodconductivityof the composite is credited to the parts evasion, strands pulling out, debonding and breakage mechanism[6].

There has been huge examinations canvases passed on for the mechanical lead and for the wear. As per the investigation wrapped up till now, it become revealed that the guide particulate graphite grows the wear rivalry and with another fortress of $\mathrm{Al} 2 \mathrm{O} 3$ in a hybrid cross section, mechanical properties where ventured forward and additionally at raised temperatures [7]. In any occasion, for the $\mathrm{Al}$ amalgam A356 and reinforcementofSiCcomposites, ithasbeeninvestigatedfor dry sliding wear considers, shows better mechanical direct stood out from the ones with out overlaying $[8,9]$. 
Fromtheliteraturesurvey,thereisalackofdataavailable for mechanical conduct of Al2219 strengthened with TiC particulates. The present work "Hardness and tractable direct of Al2219-TiC metal structure living arrangements" has been fabricated and depicted. The microstructure and the mechanical conduct of Al2219 total grid with strengthened TiC particulates had been tried. Mechanical properties likehardness(BHN)andtensilestrengthofAl2219alloyand Al2219 compound with 2, 4 and six wt. \% of $\mathrm{TiC}$ composites were surveyed in accordance with ASTMstandards.

\section{EXPERIMENTALDETAILS \& RESULTS}

\subsection{MaterialsUsed}

For the metallic system composite the base total A12219 is invigorated with Titanium carbide powder of 50-60 microns in size and is engineered as Al2219-TiC steel network composites. Thickness of Al2219 is 2.Eighty four $\mathrm{g} / \mathrm{cc}$ and that of the stronghold atom is $4.93 \mathrm{~g} / \mathrm{cc}$. The counterfeit company of A12219 base total is showed up in Table 1.

\begin{tabular}{ll}
$\begin{array}{l}\text { Table 1. Chemical composition of Al2219 alloy } \\
\text { Elements }\end{array}$ & Wt. Percentage \\
\hline $\mathrm{Si}$ & 0.20 \\
$\mathrm{Fe}$ & 0.30 \\
$\mathrm{Cu}$ & 6.8 \\
$\mathrm{Mn}$ & 0.40 \\
$\mathrm{Mg}$ & 0.02 \\
$\mathrm{Zn}$ & 0.10 \\
$\mathrm{~V}$ & 0.15 \\
$\mathrm{Zr}$ & 0.25 \\
$\mathrm{Ti}$ & 0.10 \\
& $\mathrm{Al}$ \\
\hline
\end{tabular}

\subsection{CompositePreparation}

The steel cross section composites of Al2219-TiC have been made with the guide of the use of least perplexing and greatest practical applied strategy alluded to as vortex approach or mix tossing contraption. In step with the ASTM checks of tossing approach Al2219 end up warmed to the temperatures of $730^{\circ} \mathrm{C}$ in the electric challenge warmer. The expansion of stronghold particulate TiC moved from 2 to 6 wt. \% insteps of by utilizing the utilization of mechanical blending. On account of the development in the weight level of manual particulates $\mathrm{TiC}$ porosity distortion may in addition happen at some phase in metallic system composite [10] wherein as expanding the stirringtimereducestheporositylevel[11].Thetemperature of the electric radiator became figured out how to an accuracyof

$\pm 30^{\circ} \mathrm{C}$ the utilization of a mechanized temperature controller. Degassing administrator solid hexachloroethane (C2Cl6) changed into acquainted with expel each gobbled up gas from the fluid metallic when the temperature had been come to. Sooner than the development of $\mathrm{TiC}$ particulates, mechanical blending way become completed with the assistance of zirconia included stirrer to outline a quality vortex. The speed of the stirrer is developed to end up being for 5 -eight mins at a pole beat of $250 \mathrm{rpm}$. The TiC particulates had been preheated to a temperature of $600^{\circ} \mathrm{C}$ in a preheater to expand the wettability. The stirrer wasimmersedintothemoltenmetalinthecrucibleatadepth of/3 from the base. The development of the $\mathrm{TiC}$ particulates to the fluid metal rise as apportioned into equal hundreds ratheraddingallatoncetoavoidagglomerationofthematrix. At every degree, blending changed into performed while associate of help particulate $\mathrm{TiC}$ with the fluid metal. Before discharging the fluid metal into the shape skip on, the fluid metal become warmed for around 5 minutes. The condense filled a preheated fashioned iron structure measurement of $140 \mathrm{~mm} \times 25 \mathrm{mmdiameter}$.

\subsection{Checking out}

Microstructure and mechanical lead of the Al2219 blend and its composites have been executed. A metallographic assessment changed into wrapped up by utilizing sifting electron amplifying focal point. The occasion preparation for microstructural investigate changed into completed first through cleaning the diminish models withemerypaperupto1000gritsize,followedbypolishing with $\mathrm{A} 12 \mathrm{O} 3$ suspension on a crushing gadget using velvet texture. At long last, the models had been cleaned using zero.Three microns gem stick. The cleaned off floor become scratched withKeller'sreagentandexaminedwithascanningelectron amplifying focal point. The tractable homes of the model had been imagined by methods for using a virtual versatile looking at gadget at room temperature depending on ASTM favored. Hardness tests were done on as cast Al2219 amalgam and Al2219-TiC composites to secure the impact of TiC flotsam and jetsam in the system texture. The cleaned smooth models were endeavored for their hardness, utilizing Brinell hardness looking at device havingball indenter for $250 \mathrm{~kg}$ stack and stand time of $30 \mathrm{sec} .5$ arrangements of readings have been taken at higher areas of the model and a typical unquestionably worth become used forcalculation.

\section{Outcomes and talk}

\subsection{MicrostructuralAnalysis}

Figure1(a)- (d)showstheScanningElectronMicroscope (SEM) micrographs of as steady A12219 total and its composites. Decide 1 (b) - (d) shows the SEM micrographs of 2, 4 and $6 \mathrm{wt}$. $\%$ of $\mathrm{TiC}$ particulate composites. This reveals the uniform scattering of $\mathrm{TiC}$ particles and incredibly low agglomeration and detachment of particles. The

vortex generatedinthestirringprocessbreakssoliddendritesdueto higher grinding among particles and $\mathrm{Al}$ network compound, which also turns on a uniform stream ofparticles. 


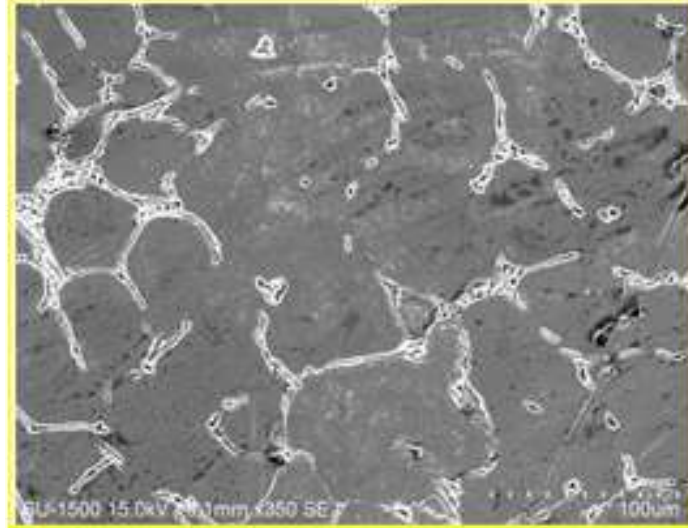

(a)

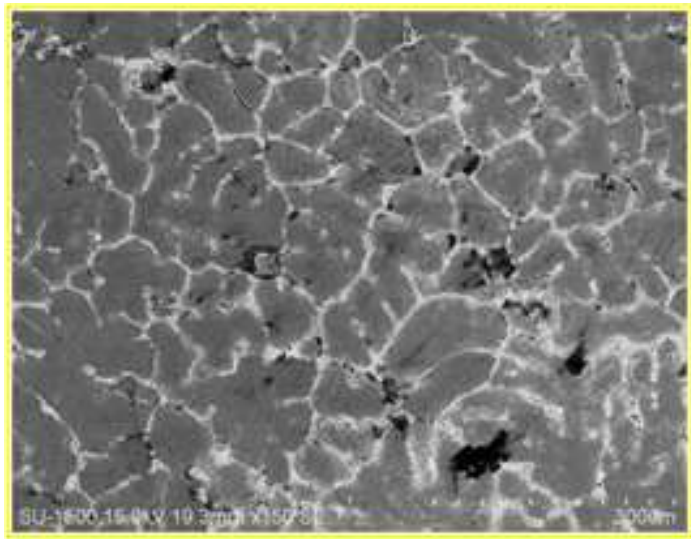

(b)

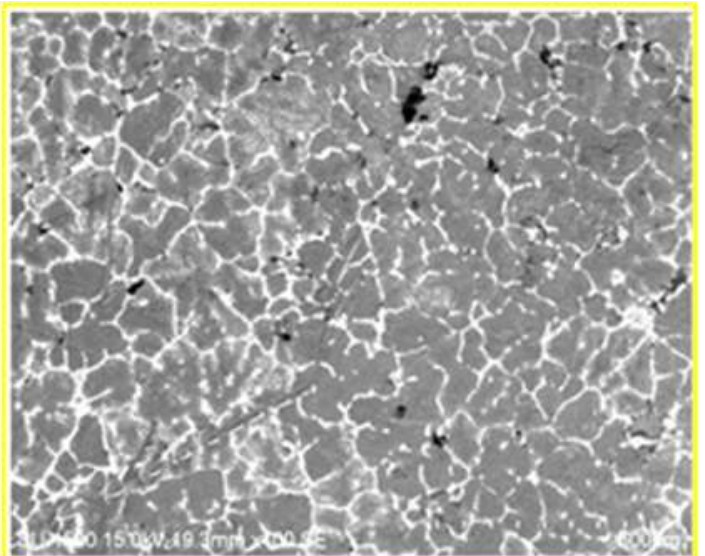

(c)

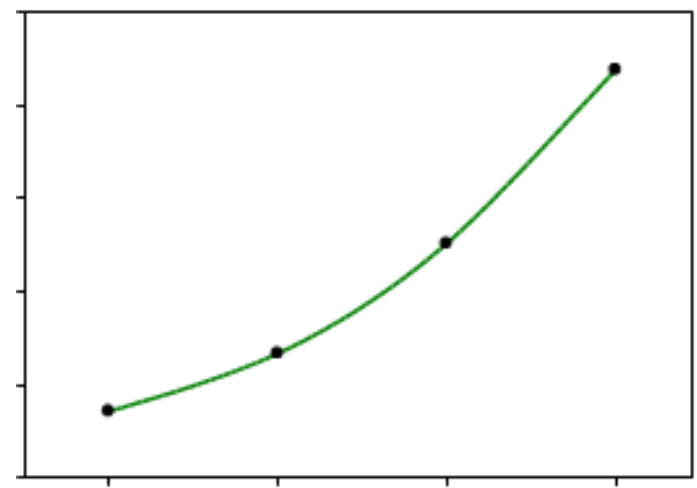

(d)

Figure 1. Showing the SEM microphotographs of (a) as cast $\mathrm{Al2219}$ alloy (b) $\mathrm{Al2219}-2 \mathrm{wt} \% \% \mathrm{TiC}$ (c) Al2219-4 wt. \% TiC (d) Al2219-6 wt. \% TiC

\subsection{HardnessMeasurements}

figure2 it, it's far located that there may be an boom in hardness Al2219 with the addition of 2, 4 and $6 \mathrm{wt} \%$ of TiC particulate.The graph showing the variation in hardness Al2219 alloy with TiC particulate reinforcement. It may be concluded that the burden advantage. \% Of TiC particulate ended in increased violence. Hardness of soft substances together with aluminum matrix reinforced with a hard growth when particulate be counted is, $\mathrm{TiC}$

Figure 2. Variation of $\mathrm{Al} 2219$ with wt. \% of $\mathrm{TiC}$ particulates in Hardness before and after addition

\subsection{Ultimate TensileStrength}

Figure three shows the tensile energy end variants (UTS) of alloy base, when reinforced with two, four and six wt. \% Of particulate TiC. Electrical closing Al2219- pull TiC composite material will be increased compared with the particulate $\quad \mathrm{TiC}$ ceramic baseA12219alloy.Themicrostructureandpropertiesofhard forged manage deformation of the composite. Due to strong bonding interface, the load of the transfer matrix for the subsequent strengthening of the power main attraction elevated. This boom in the last tensilestrengthmainlyisduetopresenceofTiCparticles

Which goes approximately as an obstruction to disengagements within the microstructure [13]. Expanded great may additionally alsobe due toalloy fortifying of thematrix precept fascination, trailed by a reduction inside the grain length of the composite, and the improvement of a excessive dislocationdensityinthe Al2219 grid compound because of the difference in heat extension between the metal network and fortification $\mathrm{TiC}$ [14].

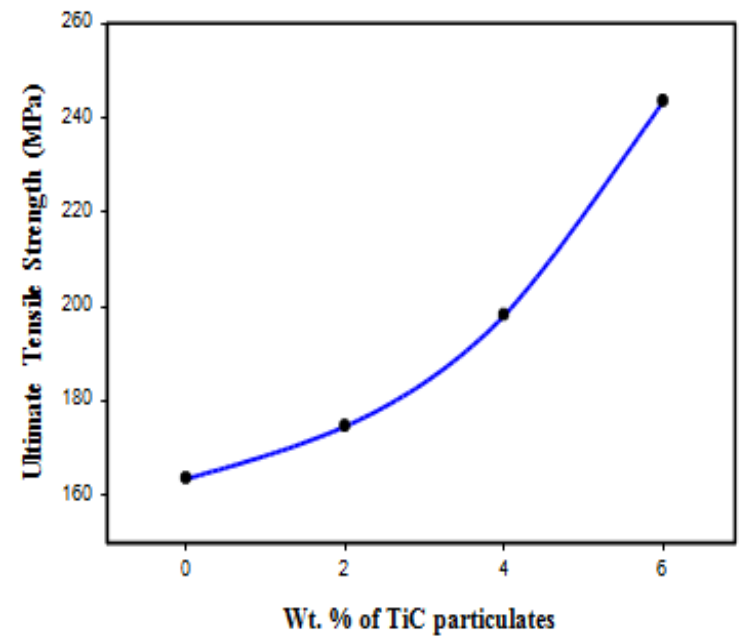

Figure 3. Variation of Al2219 with wt. \% of TiC particulates in ultimate tensile strength

1.4 YieldStrength 


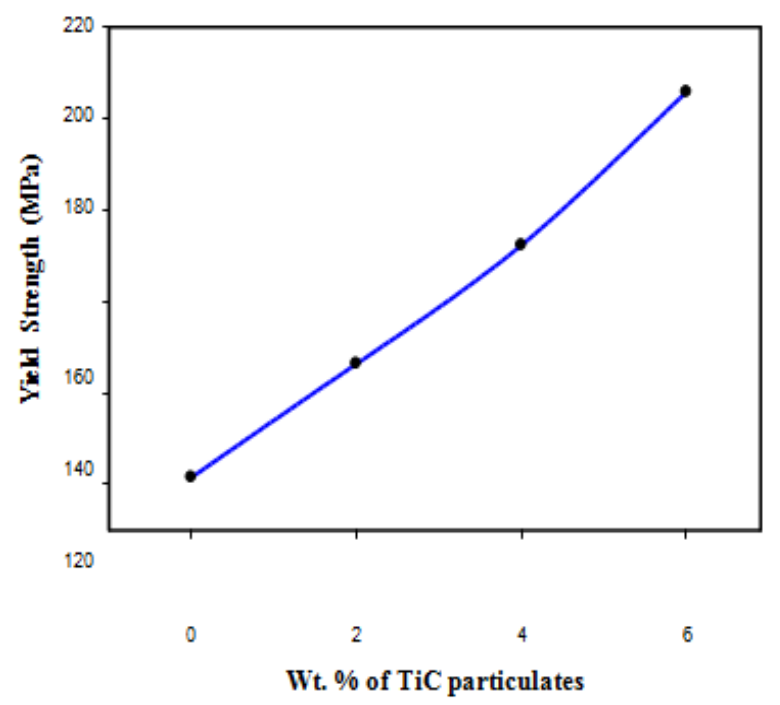

Figure 4. Variation of Al2219 with wt. \% of TiC particulates in yield strength

particles solidarity with smooth aluminum framework brings about the obstruction is more prominent than the composite against a bending load applied [15]. On account of the molecule reinforced composite, the hard particles dispersed in the lattice make-confinement to plastic flow, according to the composite provides enhanced solidarity [16].

\section{CONCLUSIONS}

Blessing works titled, "Systems Propertiesof A12219-TiC Metal Matrix Composites", has prompted the accompanying ends:

- Al2219-TiC particulate composites have been produced accurately by mixing the fluid with mind boggling throwing weight percent (ie 2, 4 and 6) ofreinforcement.

- Aluminumbasedmetalmatrixcompositeshavebeen

viably made through the method of throwing mix the fluid by methods for a two-advance expansion of fortification joined with preheating particulate.

- The thickness Al2219-2, four and 6 wt. \% TiC composites stretched out by the expansion of particulate $\mathrm{TiC}$ in A12219 basealloy.

- ImprovementsinultimatetensilestrengthoftheA12219

compound framework has been acquired by the expansion of particulate TiC. The volume increment in the combination $\mathrm{Al} 2219$ got after the expansion of two, four and six wt. \% TiC particulates have 6.Four, 21:10 and Forty Eight percent separately.

- Improved vitality of Al2219 compound framework results have been acquired with the expansion of particulate TiC. The measure of the expansion in the combination Al2219 acquired after expansion of 2, 4 and $6 \mathrm{wt}$. \% TiC particulates have 20.Forty two, and conceivably 68.Five 41.60 individually

\section{REFERENCES}

1. T. Ram Prabhu, V. K. Varma, Srikanth Vedantam, "Effect of SiC volume fraction and size on dry sliding wear of $\mathrm{Fe} / \mathrm{SiC} / \mathrm{Graphite}$ hybrid composites for high sliding speed applications", Wear, 309, pp.1-10,2014
2. Figure4 suggests sort of yield high-quality (YS) ofAl2219 compound grid with 2, four and $6 \mathrm{wt} \%$ Of TiC particulate fortified composites. It thoroughly may be visible that by using along with $6 \mathrm{wt}$. \% Of the yield satisfactory of the combination Al2219 TiC particulates multiplied from $122 \mathrm{MPa}$ to $206 \mathrm{MPa}$. The expansion inside the yield pleasant This is predictable with the effects acquired by way of certain scientists, who distinct that the quality of fortified composite particles is distinctly problem to the extent department of fortification. YS Improvement of the composite is obviously due to the nearness hardTiC

3. Zhai Wei-chen, Zhao-Hui Zhang, Fu-chi Wang, Xiangbo Shen, Shu-kui Lee, Lu Wang, "The Effect of SI content material at the microstructure and properties of $\mathrm{Si} / \mathrm{Al}$ composites", Trans. Nonferrous Met. Soc. China, 24, pp. 982 to 988.2014.

4. X.J.Wang, N.Z.Wang, L.Y.Wang, X.S.Hu, K.Wu, Y.Q Wang, Y. D. Huang, "Preparing, microstructure and mechanical living arrangements of small scale $\mathrm{SiC}$ particulate fortified magnesium grid composite phony by means of blending throwing helped with the guide of ultrasonic cure handling", Materials and Design, 59, pp.638-645.

5. H. R. Ezatpour, S. A. Sajjadi, M. H. Sabzevar, Y. Z. Huang,

"Aninvestigationofthetensileandcompressivepropertiesof Al6061 anditsnanocompositesinas-caststateandin 